\title{
Ein Dokument: Das Problem der Willensfreiheit und die Psychoanalyse
} Eine psychoanalytische Schrift des «Wolfsmanns»

Mikkel Borch-Jacobsen und Sonu Shamdasani

Der folgende Artikel ist von Sergius Constantinovitch Pankejeff, besser bekannt unter dem Namen, den ihm Freud nach einem seiner Träume gab: «der Wolfsmann». Das Leben dieses berühmten Patienten, das lange Zeit nur aus den Geschichten der Fälle bestand, die Freud ${ }^{1}$ und Ruth Mack Brunswick ${ }^{2}$ verfasst hatten, ist nunmehr besser bekannt, dank der Memoiren, die Pankejeff anonym in einem Band veröffentlichen liess, der auf Grund der Bemühungen der Psychoanalytikerin Muriel Gardiner ${ }^{3}$ herausgegeben wurde, und dank der Interviews, die er am Ende seines Lebens Karin Obholzer ${ }^{4}$ gab.

Sergius Pankejeff, 1887 bei Kherson (Ukraine) geboren, Kind ausgesprochen reicher Eltern, hatte seit seiner Jugend ein Leben der «süssen Neurose» geführt, wobei er von Spezialist zu Spezialist reiste (Bekterev, Kraepelin, Ziehen), um zu versuchen, eine unüberwindliche Melancholie und verschiedene Symptome von Obsession zu heilen. Als er 1910 in der Absicht, sich von dem Psychotherapeuten Paul Dubois in Bern behandeln zu lassen, durch Wien kam, konsultierte er Freud, der ihn dazu brachte, sich einer Analyse mit ihm zu unterziehen. Diese sollte viereinhalb Jahre dauern; Freud profitierte von ihr, dadurch dass er diesen Fall für seine Polemik gegen Jung und Adler

1 Sigmund Freud: Aus der Geschichte einer infantilen Neurose, Gesammelte Werke, London, Imgago Publishing, Bd. XII, 29-157.

2 Ruth Mack Brunswick: «A Supplement to Freud's History of an Infantile Neurosis», International Journal of Psycho-Analysis 9 (1928) 439-476.

3 Der Wolfsmann vom Wolfsmann, herausgegeben von Muriel Gardiner, Frankfurt am Main, Fischer, 1972. Dieses Buch enthält auch die von Freud und Ruth Mack Brunswick verfassten Krankengeschichten, die Kommentare von Muriel Gardiner und ein Vorwort von Anna Freud.

4 Karin Obholzer, Gespräche mit dem Wolfsmann, eine Psychoanalyse und die Folgen, Reinbek bei Hamburg, Rowohlt, 1980.

Mikkel Borch-Jacobsen, Department of Romance Languages and Literature, University of Washington, Seattle, WA 98195, USA; Sonu Shamdasani, The Wellcome Institute for the History of Medicine, 183 Euston Road, London NW1 2BE, UK 
nutzte, indem er ihn als ein Beispiel für den pathogenen Charakters des Sexuellen (oder von «Urszenen») aus der frühen Kindheit vorführte.

Freud bestand darauf, dass Pankejeff sich 1919-1920 einer zweiten Analyse unterziehe (deren Sinn dieser gar nicht einsah), anstatt nach Russland zurückzukehren, um zu versuchen, seinen Besitz vor den Bolschewiken zu retten. Als er alles verloren hatte (Freuds wegen, wie er später sagen sollte) und gezwungen war, mit seiner Frau in Wien zu bleiben, bekam er einen bescheidenen Posten als Angestellter in einer Versicherungsgesellschaft, den er bis zu seinem Ruhestand 1950 behalten sollte, wobei er gleichzeitig einige Zeit lang Geld durch eine Sammlung erhielt, die Freud unter den Psychoanalytikern angeregt hatte. Da seine Symptome aber immer noch nicht verschwunden waren,wiederholte er zwischen 1926 und 1938 noch mehrere Abschnitte der Analyse mit Ruth Mack Brunswick, einer Schülerin Freuds; in diesem Jahr traf er sich mit ihr in Paris und London, um eine schwere Depression zu heilen, die auf den Selbstmord seiner Frau gefolgt war. Nach Unterbrechung durch den Krieg sollte seine psychiatrische Karriere in Wien wieder beginnen und bis ans Ende seines Lebens dauern, unter dem Schutz von Muriel Gardiner und Kurt Eissler, die damals die Sigmund-Freud-Archive leiteten. Von Gardiner und Eissler sorgfältig vom Rest der Welt geschützt und finanziell abhängig von den Sigmund-Freud-Archiven, welche ihm jeden Monat Geld schickten, wurde Pankejeff von einer langen Reihe von Analytikern, die von seiner Identität in Kenntnis gesetzt worden waren, analysiert und/oder interviewt: Kurt Eissler (der ihn einmal am Tag während seiner Sommerferien in Wien sah), Wilhelm Solms (der ihn einmal die Woche sah), Alfred von Winterstein, Frederick S. Weil, Richard Sterba, A. Lubin und noch viele andere.

Bis 1973 musste man warten, bis es jemandem von ausserhalb des Milieus der Analytiker, der Wiener Journalistin Karin Obholzer, gelang, seine Spur wiederaufzunehmen und von ihm, nicht ohne Schwierigkeiten, Interviews zu bekommen, die nicht von seinen psychoanalytischen «Mentoren» beaufsichtigt wurden. In diesen Interviews, die nach seinem Tode veröffentlicht wurden, enthüllt der berühmte Freudsche «Wolfsmann» unter anderem, dass er niemals an die berühmte «Urszene» geglaubt habe, die Freud auf der Basis seines «Wolfstraums» rekonstruiert hatte, dass die Psychoanalyse ihm nicht im mindesten geholfen habe, sich von seiner Neurose zu befreien, und dass er sich in seinen Memoiren, die von Muriel Gardiner herausgegeben worden waren, nicht wiedererkenne. Einige Zeit nachdem er diese aufsehenerregenden Interviews gegeben hatte, verschied Sergius Pankejeff in Wien im Alter von 92 Jahren. Sein wahrer Name wurde im Jahr darauf von Kurt Eissler in seinem Bericht über die Tätigkeiten der Sigmund-Freud-Archive enthüllt 
und so endlich der Öffentlichkeit bekannt gemacht: «[Der Wolfsmann] starb am 7. Mai 1979. Sein Name, der jetzt enthüllt werden kann ...» $\gg^{5}$.

Erst 1990, elf Jahre später also, veröffentlichte Kurt Eissler den Nachlass von Sergius Pankejeff und übergab ihn der Kongressbibliothek in Washington. Diese wichtige Sammlung, die insbesondere die Familienphotographien und die Gemälde von Sergius Pankejeff enthält (der manchmal mit «Wolfsmann» unterzeichnete), birgt viele Überraschungen. Mancher mag deshalb erstaunt sein, zu erfahren, dass dieser Patient Freuds, über den so viele Kommentare verfasst wurden, selbst Autor psychoanalytischer Schriften war. In Wahrheit ist Sergius Pankejeff jedoch nicht allein jener zur Mitarbeit bereite Patient, den Kurt Eissler, Muriel Gardiner und so viele andere Psychoanalytiker unermüdlich nach seiner Erinnerung befragten, sondern lange Zeit auch ein sehr treuer und sehr orthodoxer Schüler Freuds - vielleicht der am meisten unbemerkt gebliebene in der gesamten Geschichte der psychoanalytischen Bewegung. Die zahlreichen Manuskripte und Aufzeichnungen aus seinem Nachlass sowie andere erst kürzlich entdeckte Dokumente legen Zeugnis davon ab, dass Sergius Pankejeff tatsächlich eine psychoanalytische Erklärung für so verschiedene Bereiche wie die Freiheit des Menschen, den Marxismus, Kunst, Astrologie oder die Träume Swanns in der Recherche von Proust versucht hat, und zwar in einem Stil, der, obwohl angestrengt und wenig originell, so doch völlig zusammenhängend ist und Freuds Theorie bis ins Letzte folgt.

Warum hat man nun so lange warten müssen, bis diese psychoanalytischen Texte eines der wichtigsten Patienten Freuds zugänglich wurden? Es ist zumindest merkwürdig, dass Kurt Eissler und Muriel Gardiner, obwohl beiden einige seiner Schriften seit Beginn der fünfziger Jahre bekannt waren, sich nicht einmal darum bemüht haben, diese Texte dem Publikum zugänglich zu machen, da sie doch bereit waren, seine Korrespondenz mit Freud ${ }^{6}$ und seine Memoiren zu veröffentlichen (die ärztliche Schweigepflicht kann bei dieser Entscheidung keine Rolle gespielt haben, denn es wäre leicht möglich gewesen, diese Texte unter dem Namen «Wolfsmann» zu veröffentlichen). Zweifelsohne haben sie bedacht, dass der Wolfsmann dem Anliegen der Psychoanalyse nur als Patient nützlich sein würde, nämlich passiv; dass es unangenehm, ja sogar gefährlich für sie sein würde, ihm Stimme und Feder als Autor und Akteur zu gewähren (die Versuche, die Eissler und Gardiner unternahmen, Pankejeff davon abzuhalten, Karin Obholzer ein Interview zu ge-

5 Kurt R. Eissler: «Report on the Sigmund Freud Archives», 144th Bulletin of the International Psychoanalytic Association, International Journal of Psycho-Analysis 61 (1980) 104-105.

6 «Letters pertaining to Freud's History of an Infantile Neurosis», Psychoanalytic Quarterly 26 (1957) 449-460. 
ben, sind in dieser Hinsicht nur allzu verräterisch). Vielleicht haben sie es jedoch auch für klüger gehalten, kein allzu grelles Licht auf den regelrechten Indoktrinationseffekt zu werfen, den die Psychoanalyse an einem der berühmtesten Patienten Freuds vollbracht hat:worin besteht denn eigentlich der Unterschied zwischen ihnen und ihm, zwischen den Schülern und dem Patienten?

Welcher Art ihre Motive auch immer gewesen sein mögen, sie sind für uns heute unerheblich, jetzt, da Sergius Pankejeff tot ist und seine Texte ohne Eigentümer sind. Der von uns im Anschluss veröffentlichte Text ist aus einem Band entnommen, der die psychoanalytischen Schriften von Sergius Pankejeff versammeln soll und der demnächst in englischer und französischer Übersetzung erscheint. So wird man sich schnell versichern können, dass dieser Text, der offensichtlich im Hinblick auf eine Veröffentlichung verfasst wurde, den meisten anderen psychoanalytischen Veröffentlichungen der vierziger und fünfziger Jahre an Originalität weder nachsteht noch sie darin übertrifft (das gleiche könnte man auch von den anderen psychoanalytischen Schriften Sergius Pankejeffs sagen). Deshalb muss hervorgehoben werden, dass dieser Artikel vor allem von dokumentarischem und historischem Interesse ist. Der Text dieser Edition wurde nach dem maschinengeschriebenen Originalmanuskript erstellt, das Kurt Eissler 1990 in der Kongressbibliothek in Washington hinterlegt hat. Der Zeitpunkt, zu dem der anschliessend reproduzierte Text verfasst wurde, ist ungewiss. Verschiedenes deutet jedoch darauf hin, dass dieser Text vor 1953 entstand und wahrscheinlich gegen Ende der vierziger oder zu Beginn der fünfziger Jahre verfasst wurde. Die Fussnoten haben wir angefügt. 